\title{
Application Effect of Predictive Nursing in the Nursing Process of Patients with Coronary Heart Disease and Angina Pectoris
}

\author{
$\mathrm{Yi} \mathrm{Lu}^{1}$ \\ ${ }^{1}$ Henan Province Hospital of Traditional Chinese Medicine Zhengzhou, Henan 450000
}

\begin{abstract}
To explore the measures and clinical effects of applying predictive nursing methods in the process of nursing care of patients with coronary heart disease and angina pectoris. The patients who were treated in our hospital from December 2019 to December 2020 were selected as the research objects. The patients were all with coronary heart disease and angina pectoris. Nursing care of the patients was carried out. 100 patients were randomly selected as the research objects. In the controlled experiment, patients were given conventional nursing intervention (control group) and predictive nursing intervention (experimental group), and the clinical intervention effects of the two nursing methods were compared and analyzed. The number and duration of angina pectoris in the experimental group were lower than those in the control group. The nursing satisfaction of the two groups was $96.00 \%$ and $82.00 \%$ respectively. The satisfaction of the patients in the experimental group was higher, and the blood pressure and blood sugar of the experimental group were normal. The number of normal blood lipids was higher than that of the control group. The SAS score and SDS score of the experimental group were relatively low compared with the control group, and the differences in the data were large, expressed as $\mathrm{P}<0.05$, which was statistically significant. The effect of the experimental group is better. Applying predictive nursing methods in the process of nursing care for coronary heart disease and angina pectoris has significant clinical effects, which can effectively stabilize various physical indicators of the patient community, improve patient care satisfaction, and have significant clinical application value.
\end{abstract}

\section{Introduction}

Patients with coronary heart disease usually have angina pectoris. The clinical pathogenesis of the patients is not clear. It is believed to be caused by a variety of factors. According to relevant research data, the incidence of diabetes, hyperlipidemia and hypertension is higher in patients, which are the main factors to affect the coronary heart disease and angina pectoris ${ }^{[1]}$. In recent years, the incidence of coronary heart disease patients has gradually shown an upward trend, posing a serious threat to the lives and health of patients. When giving patients treatment, in order to ensure the clinical effect, it is also necessary to cooperate with nursing methods to promote patients to maintain a good attitude and reduce the incidence of adverse reactions ${ }^{[2]}$. Based on this, this study taking patients in our hospital as an example, applying predictive nursing methods to explore its clinical application value.

\section{Information and methods}

\subsection{General information}

The location of this research has been chosen in our hospital. The research will be carried out from December 2019 to December 2020. The patients are all patients with coronary heart disease and angina pectoris receiving treatment during this period. 100 cases are selected and the patients are divided into two groups, as the experimental group and the control group, each group corresponds to a different nursing method, and the number of patients is 50 cases. In the experimental group, the ratio of the number of males to females is $26: 24$. The maximum age of the patients is 87 years old, the minimum age is 54 years old, and the average age of the patients is $(63.23 \pm 5.40)$ years old. In the control group, the ratio of males and females is $27: 23$, the maximum and minimum ages of patients are 88 and 53 years, and the average age is $(64.03 \pm 5.44)$ years. The data of the two groups of patients have little difference, and comparative experiments can be realized.

\subsection{Research methods}

The patients in the control group were given conventional nursing methods, and the patients were regularly monitored by electrocardiogram, and the patients were informed of the necessity of the development of treatment work, and the patients' diet was controlled.

Firstly, patients in the experimental group adopt predictive care. In the specific implementation process, patients are first given predictive care with legal factors. Under normal circumstances, patients will be affected by various factors such as postural changes, emotional self, movement and sleep disorders, and diet changes, whcih 
will cause patients to develop coronary heart disease and angina pectoris, so nursing staff need to inform patients of the main factors that cause angina pectoris, regulate the patient's life and rest, ensure that patients develop good living habits, reduce the incidence of angina, protect the cardiovascular system, and reduce external stimuli. Secondly, patients are given psychological predictive care. Coronary heart disease and angina pectoris are prone to recurring attacks, and the disease is characterized by sudden onset. In this case, the patient is prone to various negative emotions, and it is necessary for nursing staff to grasp the patient's negative emotions and communicate with the patients, formulate personalized psychological care plans for the patients, answer the patients' questions, and explain the occurrence of diseases, treatment methods and prognostic effects, etc., to improve the patients' awareness and reduce unnecessary worry, and ensure the patients to actively cooperate with the treatment work. At the same time, the patients are provided with predictive care for the observation of their condition. During the treatment process, the patients' vital signs are strictly monitored, the nature, degree and duration of the patients' pain are selected, and the appropriate pain relief method is selected for the patients. When the patients are hospitalized, it is necessary to strengthen the inspection of the ward. Once the patients are found to have angina pectoris symptoms, it is necessary to give the patients nitroglycerin immediately and instruct the patients to take it under the tongue, so as to ensure the safety of the patients' life. Finally, physical and mental relaxation training requires nurses to inform the necessity of the development of the physical and mental training, and guide the patients to start the training, explain the training method, help the patients choose an appropriate posture, try to use sitting or lying down as much as possible to promote the patients' body muscles in a relaxed state, nursing staff should give the patients massage to relax, reduce the patients' psychological burden, and promote the patients' recovery.

\subsection{Observation indicators}

The number of angina pectoris episodes and duration of the two groups of patients were observed and recorded, and statistical analysis was performed.

To evaluate the nursing satisfaction of the two groups of patients, we used our hospital's self-made satisfaction survey. The indicators included three items, namely very satisfied, satisfied and dissatisfied. The questionnaire has a total score of 100 . When the score is 80 or above, it is very satisfied, when the score is between 60 and 80 , it is satisfied, and when the score is less than 60, it is dissatisfactory. Exclude dissatisfaction is the total satisfaction rate.

The physical signs of the two groups of patients after treatment were monitored, and the number of people with normal blood sugar, normal blood lipids and normal blood pressure was recorded and compared.
The physical signs of the two groups of patients after treatment were monitored, and the number of people with normal blood sugar, normal blood lipids and normal blood pressure was recorded and compared.

The depression self-rating scale and the anxiety selfrating scale are used to evaluate the anxiety and depression of the two groups of patients. A high score indicates that the patient's depression and anxiety are serious [3].

\subsection{Statistical methods}

Use statistical software SPSS20.0 as a tool to perform statistical analysis on the data appearing in this study, verifying the comparison results of measurement data $(\mathrm{x} \pm \mathrm{s})$ with $t$ value, and verifying the count data $(n, \%)$ by the $x 2$ value. When the results showed $\mathrm{P}<0.05$, it indicated that the differences between the groups had statistical analysis value.

\section{Results}

\subsection{Angina pectoris}

The number and duration of angina pectoris in the experimental group were $(0.87 \pm 0.34)$ times and $(4.55 \pm 1.23) \mathrm{min}$ respectively, which were lower than those of the control group. There were significant data differences between the groups, $\mathrm{P}<0.05$.

Table1. Comparison of the number and duration of angina pectoris between the two groups $(\mathrm{x} \pm \mathrm{s})$

\begin{tabular}{ccc}
\hline Group & $\begin{array}{c}\text { Number of } \\
\text { episodes } \\
\text { (times) }\end{array}$ & $\begin{array}{c}\text { Duration } \\
(\mathbf{m i n})\end{array}$ \\
\hline $\begin{array}{c}\text { Experimental } \\
\text { group } \\
(\mathbf{n = 5 0})\end{array}$ & $0.87 \pm 0.34$ & $4.55 \pm 1.23$ \\
$\begin{array}{c}\text { Control group } \\
(\mathbf{n = 5 0})\end{array}$ & $2.34 \pm 0.55$ & $8.67 \pm 2.31$ \\
$\mathrm{t}$ & 5.445 & \\
& & 6.056 \\
$\mathrm{P}$ & $<0.05$ & $<0.05$ \\
\hline
\end{tabular}

\subsection{Two groups of patients care satisfaction}

The nursing satisfaction of the two groups of patients were $96.00 \%$ and $82.00 \%$, respectively. The patients in the experimental group were more satisfied with the data difference, $\mathrm{P}<0.05$, which is meaningful. 
Table2. Comparison of nursing satisfaction scores between the two groups of patients [n(\%)]

\begin{tabular}{ccccc}
\hline Group & Very satisfied & Satisfied & Dissatisfied & Satisfaction \\
\hline $\begin{array}{c}\text { Experimental group } \\
(\mathbf{n}=\mathbf{5 0})\end{array}$ & $36(72.00)$ & $12(24.00)$ & $2(4.00)$ & $48(96.00)$ \\
$\begin{array}{c}\text { Control group } \\
(\mathbf{n = 5 0})\end{array}$ & $23(46.00)$ & $18(36.00)$ & $9(18.00)$ & $41(82.00)$ \\
$\mathrm{X}^{2}$ & & & & 7.697 \\
$\mathrm{P}$ & & & $<0.05$ \\
\hline
\end{tabular}

\subsection{Body indicators after intervention}

The number of patients with normal blood sugar, normal blood pressure and normal blood lipids in the experimental group was higher than that in the control group, and the difference was statistically significant $(\mathrm{P}<0.05)$.

Table3. Analysis of the body-related situation of the two groups of patients after intervention $[\mathrm{n}(\%)]$

\begin{tabular}{cccc}
\hline Group & $\begin{array}{c}\text { Normal } \\
\text { blood sugar }\end{array}$ & $\begin{array}{c}\text { Normal } \\
\text { blood } \\
\text { pressure }\end{array}$ & $\begin{array}{c}\text { Normal } \\
\text { blood lipids }\end{array}$ \\
\hline $\begin{array}{c}\text { Experimental } \\
\text { group (n=50) }\end{array}$ & $40(80.00)$ & $41(82.00)$ & $43(86.00)$ \\
$\begin{array}{c}\text { Control group } \\
(\mathbf{n = 5 0})\end{array}$ & $19(39.00)$ & $20(40.00)$ & $17(34.00)$ \\
$\mathrm{X}^{2}$ & 4.956 & 6.005 & 5.486 \\
& $<0.06$ & $<0.06$ & $<0.06$ \\
$\mathrm{P}$ & & & \\
\hline
\end{tabular}

\subsection{Psychological and emotional scores of the two groups of patients}

Compared with the control group, the SAS score and SDS score of the experimental group were relatively low, and the data difference between each group was large, $\mathrm{P}<0.05$, and the mental state of the experimental group was good.

Table4. Comparison of psychological and emotional scores between the two groups $(\mathrm{x} \pm \mathrm{s})$

\begin{tabular}{ccc}
\hline Group & SAS & SDS \\
\hline $\begin{array}{c}\text { Experimental } \\
\text { group (n=50) } \\
\begin{array}{c}\text { Control group } \\
(\mathbf{n = 5 0})\end{array}\end{array}$ & $43.23 \pm 1.22$ & $36.56 \pm 3.23$ \\
$\mathrm{t}$ & 10.003 & \\
$\mathrm{P}$ & $<0.05$ & $85.956 \pm 4.05$ \\
\end{tabular}

\section{Conclusion}

Angina pectoris is a common type of coronary heart disease. It is mainly caused by coronary artery atherosclerosis, which leads to stenosis of the official cavity, which leads to coronary artery spasm and myocardial ischemia. The patient shows paroxysmal pain and compressive pain in the front chest, which will have a great impact on the quality of life and health of patients ${ }^{[4]}$. In recent years, the incidence of patients with coronary heart disease and angina pectoris has been increasing. Most of the patients are elderly. They are affected by age factors and have a decline in immune function, which has adverse effects on patients. It is necessary to clinically strengthen the treatment of patients and pay attention to the degree and give patients active care ${ }^{[5]}$.

Predictive nursing is a new type of nursing model. It starts from the patient's own physical condition and the characteristics of the disease, and takes measures according to the patient's condition, which will reduce the risk of nursing, and provides patients with more professional and humanized nursing services to ensure patients' satisfaction and quality of care ${ }^{[6]}$. For patients with coronary heart disease and angina pectoris, it is necessary to do predictive care for their changes. It is also necessary to strengthen diet intervention, provide patients with psychological care, and carry out health education, so as to ensure that patients receive treatment with good attitude, reduce the incidence of adverse reactions and increase patient's treatment compliance in order to develop good living habits, ensure patients to have adequate sleep, reasonable diet, achieve effective control of the disease, and ensure the treatment effect ${ }^{[7]}$. After the application of predictive nursing methods in this study, the results showed that the patients in the experimental group had higher nursing satisfaction, the incidence of angina pectoris was low, the duration was short, and the incidence of unhealthy psychology of the patients was low, and various physical indications were better. The data are significantly different from those in the control group $(\mathrm{P}<0.05)$. It shows that the predictive nursing method has higher application value in patients with coronary heart disease and angina pectoris.

In summary, the application of predictive nursing methods in patients with coronary heart disease and angina pectoris has significant clinical effects. It can reduce the incidence of angina pectoris in patients and ensure that the vital signs of patients are significant and can be promoted in clinical practice. 


\section{Acknowledgement}

As this paper is about to be completed, I would like to express my gratitude to the people who provided help in writing this paper. Thank you for your guidance on my paper. With your help, my paper can be presented in a more professional state. I am here to express my sincere thanks and wish all good health.

\section{Reference}

1. Shen Yanju, Lin Jialin, Zhang Yingshun. The effect of predictive nursing in the nursing process of patients with coronary heart disease and angina pectoris[J]. Electronic Journal of Practical Clinical Nursing, 2019, 004(022): P.84-85.

2. Tang Zhengying. Observation on the application effect of predictive nursing in the nursing of patients with coronary heart disease and angina pectoris[J]. Electronic Journal of Integrated Traditional Chinese and Western Medicine Cardiovascular Diseases, 2019, 007(001):152-153.

3. Yu Li. Application analysis of predictive nursing in the care of patients with coronary heart disease and angina pectoris $[\mathrm{J}]$. Chinese Medical Guide, 2020, 018(006):362-363.

4. Lu Xiaoqin. Observation on the effect of predictive nursing in the nursing of patients with unstable angina pectoris[J]. Diet Health Care, 2019, 6(002):144-144.

5. Li Zengyue. Analysis of the effect of predictive nursing in the nursing of patients with coronary heart disease and angina pectoris $[\mathrm{J}]$. Friends of Health, 2020, 000(007):178.

6. Yang Lianjuan, Xiao Fengqin. Analyze the application effect of predictive nursing procedures in the nursing of inpatients with coronary heart disease[J]. Health for All, 2020, No.523(14):438-438.

7. Zhao Xin, Li Panpan. Application effect of predictive nursing in primary preventive nursing intervention for young patients with coronary heart disease[J]. Laboratory Medicine and Clinics, 2019(13). 